Research Article

\title{
Effects of the Amount of Fly Ash Modified by Stearic Acid Compound on Mechanical Properties, Flame Retardant Ability, and Structure of the Composites
}

\author{
Tuan Anh Nguyen \\ Department of Chemical Technology, Hanoi University of Industry (HaUI), No. 298 Cau Dien, North District Tu Liem, \\ Hanoi, Vietnam
}

Correspondence should be addressed to Tuan Anh Nguyen; anhnt@haui.edu.vn

Received 3 July 2019; Accepted 7 January 2020; Published 1 March 2020

Academic Editor: Donald L. Feke

Copyright $\odot 2020$ Tuan Anh Nguyen. This is an open access article distributed under the Creative Commons Attribution License, which permits unrestricted use, distribution, and reproduction in any medium, provided the original work is properly cited.

Fly ash, a waste product from thermal power plants, is one of the good alternatives for use as a filler in polymers, especially in flame retardants. Fly ash is an environmentally friendly fire retardant additive for composites, used in place of conventional flame retardant additives such as halogenated organic compounds, thus promoting environmental safety. In this study, fly ash was modified with stearic acid to improve adhesion at the polymers interface and increase compatibility. Fly ash was studied at various volumes $(5,10$, and $20 \mathrm{wt} . \%$ fly ash) used in this study to synthesize fly ash-epoxy composites. The results show that the tensile strength, flexural strength, compressive strength, and impact strength of these synthetic materials increase when fly ash is modified to the surface, compressive strength: $197.87 \mathrm{MPa}$, flexural strength: $75.20 \mathrm{MPa}$, impact resistance: $5.77 \mathrm{KJ} / \mathrm{m}^{2}$, and tensile strength: $47.89 \mathrm{MPa}$. Especially, the fire retardant properties are improved at a high level, with a modified $20 \%$ fly ash content: the burning rate of $16.78 \mathrm{~mm} / \mathrm{min}$, minimum oxygen index of $23.2 \%$, and meet the fire protection standard according to UL 94HB with a burning rate of $8.09 \mathrm{~mm} / \mathrm{min}$. Scanning electron microscopy (SEM) and infrared spectroscopy were used to analyze the morphological structure of fly ash after being modified and chemically bonded with epoxy resin background.

\section{Introduction}

Fly ash, nearly $40 \%$, remain unused and were left at the plant and eventually will intentionally cause environmental pollution [1]. A significant advantage is if this massive leftover material will be used in other areas of application that may have positive influences on the production cost and environment. Fly ash seems to have a huge potential in terms of utilization as tonnes of this byproduct is left unused at the power plant or dumped at the landfill site.

Thus, researchers are always finding a way to maximize the utilisation of FA in various applications. In recent years, the trend has been focusing on the discovery of a new or recyclable material that can be integrated in polymers. The purpose is to replace existing materials that have been prohibited due to environmental and health constraints without compromising mechanical properties but maintaining its characteristic [2].

Polymer matrix composites are used in greatest diversity in light of their less cost, ease of fabricability, higher specific strength, design flexibility, and lightweight. In fibre epoxy composites, the addition of fly ash led to a reduction of the density and increase in modulus of composites [3]. Vipulanandan et al. $[4,5]$ investigated epoxy- and polyester-based polymer mortars, and they determined that the optimal quantity of resin is between $14 \%$ and $16 \%$. Singla \& Chawla studied epoxy-based composites including fly ash [6].

\section{Materials and Methods}

2.1. Materials. Dietylentriamine (DETA), Dow Chemicals (USA). EPIKOTE Resin 240 of Dow Chemicals (USA) is a low-viscosity epoxy resin based on a blend of a bisphenol A 
resin (produced from bisphenol A and epichlorohydrin) and a bisphenol $\mathrm{F}$ resin (produced from bisphenol $\mathrm{F}$ and epichlorohydrin), containing an added proportion of a monoepoxidised alcohol as a reactive diluent.

Fly ash taken from the ash waste of Pha Lai Thermal Power Station was collected in Song Da Joint Stock Company 12- Cao Cuong, Vietnam. Before investigation, fly ash was washed by acetone to remove impurities.

2.2. Specimen Preparation. Both the epoxy and fly ash (FA) were dried for $1 \mathrm{~h}$ at $80^{\circ} \mathrm{C}$ under vacuum prior to blending 5 ; 10 and 20 wt. $\%$ FA was dispersed in epoxy at $80^{\circ} \mathrm{C}$, using a mechanical stirrer at about 3000 revolutions per minute $(\mathrm{rpm})$ for $3 \mathrm{hr}$. Then, the blends were degassed under vacuum for 15-20 min inside an oven and poured into an aluminium mould and cured for $3 \mathrm{~h}$ at $80^{\circ} \mathrm{C}\left( \pm 2^{\circ} \mathrm{C}\right)$. After 7 days of removing the sample, it was taken to measure the substance.

2.3. Characterization and Testing. Mechanical tests were conducted on at least five specimens by a $100 \mathrm{kN}$ universal testing machine (INSTRON-5582, USA). Flexural properties were determined by using three-point bending test specimens with dimensions of $100 \times 15 \times 4 \mathrm{~mm}$ according to ISO 178. Compressive properties were determined by using three-point bending test specimens with dimensions of $15 \times 10 \times 10 \mathrm{~mm}$ according to ISO 178-1993. Izod Impact Strength was determined according to ASTM D265 satndard in Tinius Olsen (USA). Tensile properties were determined by using three-point bending test specimens according to ISO 178.

Limiting oxygen index values were measured by an oxygen index apparatus (Fire Testing Technology, Japan), having a paramagnetic oxygen analyzer so that precise adjustments of the oxygen concentration can be performed, and repeatable results are obtained. Tests were conducted on bars with dimensions of 70 to $150 \times 6.5 \pm 0.5 \times 3 \pm 0.5 \mathrm{~mm}$ at relative humidity of $40 \%$. Oxygen concentrations were varied according to Dixon's up-and-down procedure explained in JIS K7201. The method entails the use of a large number of specimens of the same type to determine oxygen index with $\sim 0.15 \% \mathrm{O}_{2}$ of standard deviation. The Horizontal Burning tests (UL-94HB): standard bar specimens are to be $125 \pm 5 \mathrm{~mm}$ long by $13.0 \pm 0.5 \mathrm{~mm}$ wide and provided in the minimum thickness and $3.0(-0.0+0.2) \mathrm{mm}$ thick. (ASTM D635-12).

\section{Results and Discussion}

\subsection{Treating the Fly Ash Surface by Stearic Acid}

3.1.1. Analysis of IR Spectroscopy of Fly Ash Modified with Stearic Acid. Stearic is a saturated fatty acid C17 including active carboxyl group. In the standard conditions, stearic acid is a solid acid with white, light, spherical particles (density of 0,893 ) like the structure of fly ash ones. Stearic acid particles were dissolved in a mixture of toluene and acetone solutions. The amount of stearic acid used for modification depends on the amount of fly ash.

We qualitatively evaluate the efficiency of this method through peaks of groups in IR spectra of modified fly ash. The amount of stearic acid used for the modification was $6 \%$ (3 $\mathrm{g}$ stearic acid for $50 \mathrm{~g}$ fly ash). IR spectra of fly ash modified with stearic acid are shown in Figure 1.

In Figure 1, there are new peaks at $3445 \mathrm{~cm}^{-1}, 2914 \mathrm{~cm}^{-1}$, $1705 \mathrm{~cm}^{-1}$, and $1385 \mathrm{~cm}^{-1}$ in comparison to initial fly ash. The broad peak at $3445 \mathrm{~cm}^{-1}$ corresponds to $-\mathrm{OH}$ groups of carboxyl acid, and the peak at $2914 \mathrm{~cm}^{-1}$ corresponds to stretching vibration of $\mathrm{C}-\mathrm{H}$ bonds of stearic acid. Besides, the peaks at $1705 \mathrm{~cm}^{-1}$ and $1385 \mathrm{~cm}^{-1}$ correspond to stretching vibration of $\mathrm{C}=\mathrm{O}$ bonds and $\mathrm{C}-\mathrm{O}$ bonds in carboxyl groups of stearic acid, respectively, proving the presence of stearic acid on the fly ash surface. So, it can be said that stearic acid interacted with fly ash particles.

3.1.2. Effects of Stearic Acid Treatment on the Contribution of Fly Ash's Size and Surface Area. Fly ash was mixed with stearic acid to modify its surface, then filtered and dried at $100^{\circ} \mathrm{C}$, and stabilized in a desiccator within 1 day. The modified fly ash's surface was observed by the SEM method.

SEM images in low resolution (Figure 2(b)) shows that fly ash particles modified with stearic acid are equal in size and even in contribution, while there is an irregularity in both size and contribution for initial fly ash particles (Figure 2(a)). Besides, SEM images in high resolution (Figure 2(d)) show that even though modified fly ash particles are not broken to release smaller inside particles, the surface is so rough and stearic acid does break the structure of fly ash but cover fly ash particles' surface. For that reason, modification with stearic acid makes the contact ability as well as adhesion between fly ash and epoxy resin substrate better. Besides, fly modified with stearic acid also makes it easier for fly ash to disperse into resin.

\subsection{Effect of Fly Ash Treated with Stearic Acid to Epoxy-Based} PC Material Structure. Through different resolutions, it was observed that the fly ash particle after denaturing with stearic acid has better adhesion and compatibility in base resin by a rough contact surface. A better fly ash and substrate are shown in Figure 3. In addition, the fly ash particle-modified stearic acid also dispersed well in the resin base, and the density of fly ash particles in the sap is quite large (Figure 3).

3.3. Effects of the Amount of Fly Ash on Mechanical Properties of the Materials. See Figure 4; through the abovementioned figures, after being modified with stearic acid, the mechanical strength of the fly ash is increased, although the impact strength, tensile strength, and flexural strength are reduced when the fly ash content increases but not significant. And the plastic sample with fly ash wt.\% 20 is the most optimal because it has the most fly ash reinforcement, and the mechanical strength is not affected too much, reducing the cost of products for practical application. 


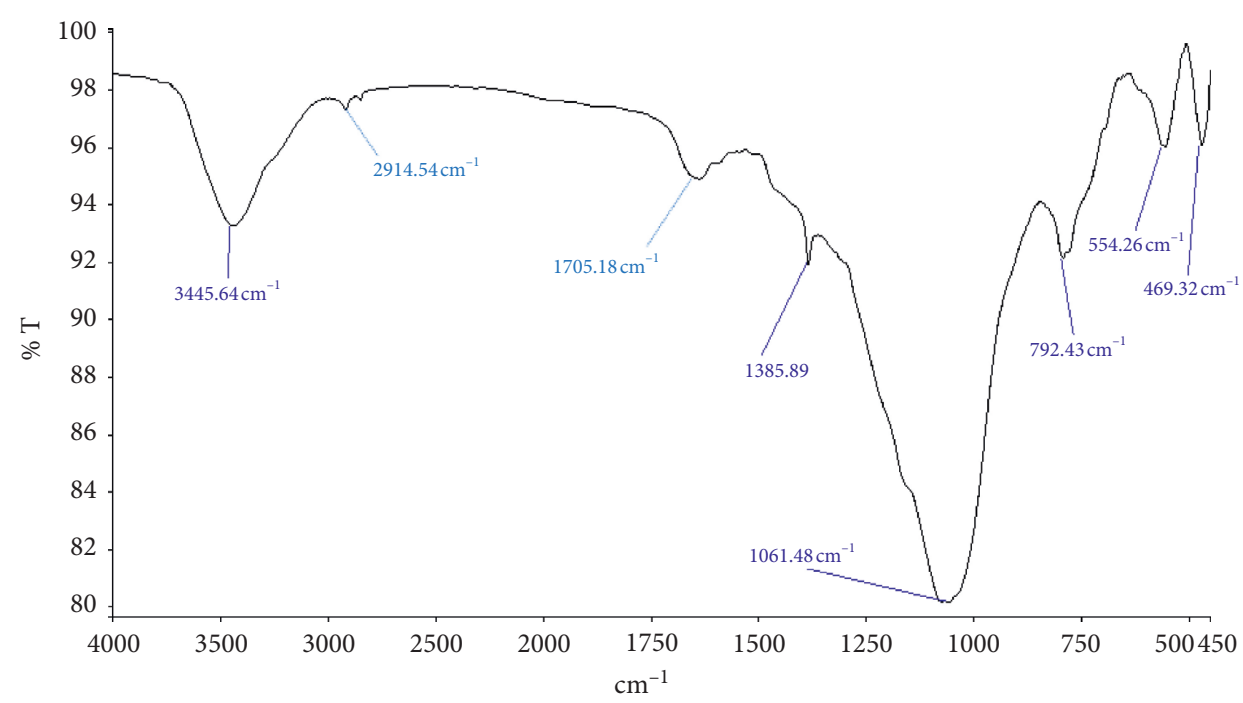

FIGURE 1: IR spectrum of fly ash samples-modified stearic acid.

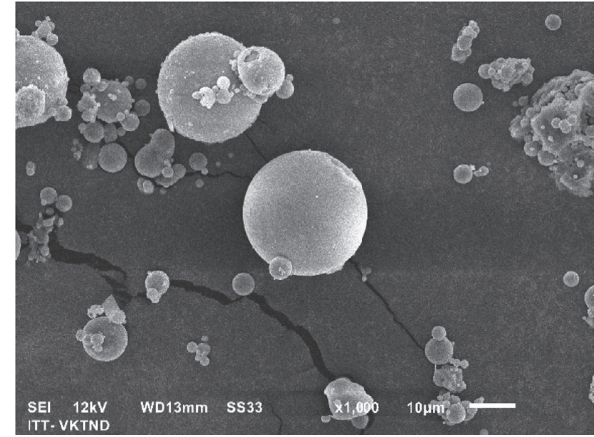

(a)

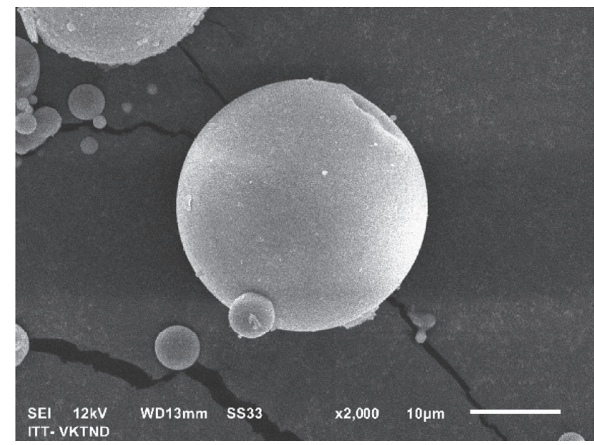

(c)

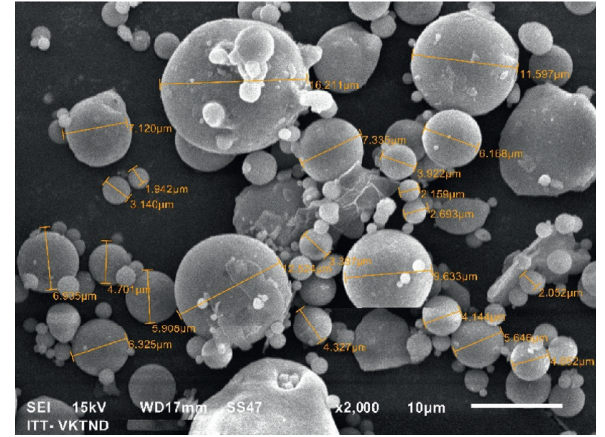

(b)

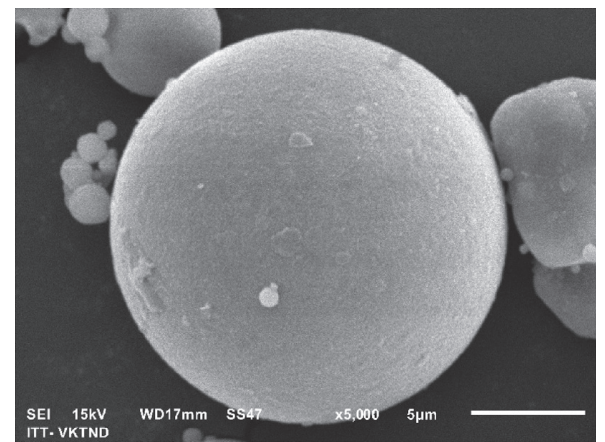

(d)

FIgUre 2: SEM image of fly ash-modified stearic acid: (a, d) fly; (b, c) fly ash modified by stearic acid.

When epoxy resin cures with diethylenetriamine (DETA), forming cross-linking, it forms a three-dimensional space circuit. Fly ash acts as an inorganic filler that loses the space between polymer chains, reducing the flexibility of the polymer molecule to increase hardness.

At the same time, the particles fly ash into the pores in the matrix, and they squeeze the polymer in the vicinity, reducing tension and increasing hardness. In addition, the adhesion of the particle/matrix has a significant influence on the durability of granular polymer composites. A strong interfering link between particles and polymer matrix is important for effective stress transfer resulting in high synthesis intensity (See Figure 5).

3.4. Effects of the Amount of Fly Ash on Flame Retardant Properties of the Materials. Figure 6 shows that burning rate is lowest $(8.41 \mathrm{~mm} / \mathrm{min})$ for sample including $20 \mathrm{wt} \%$ of fly ash. Therefore, increasing the amount of fly ash decreases flammability and burning rate of materials. 

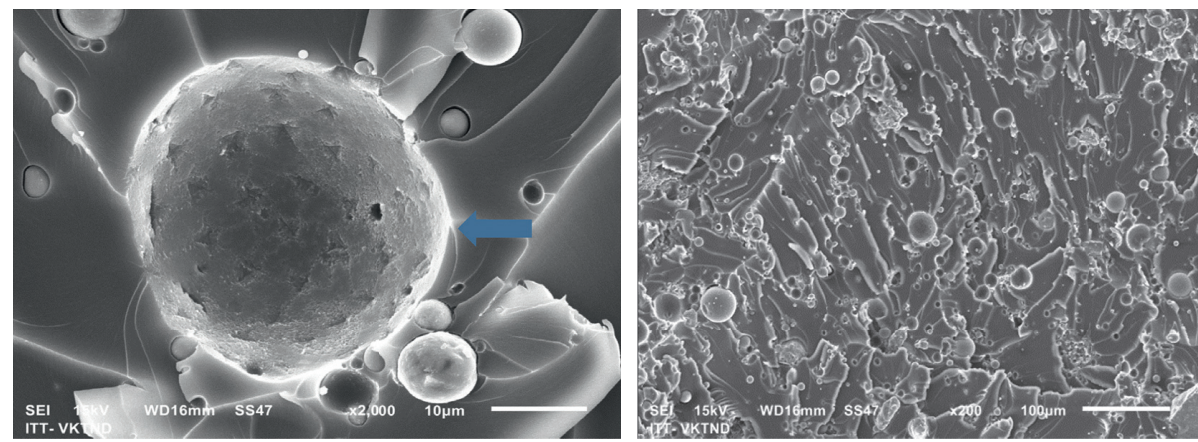

FIGURE 3: SEM images of composite materials reinforced fly ash stearic acid denatured $\times 2000 ; \times 200$ times resolution.
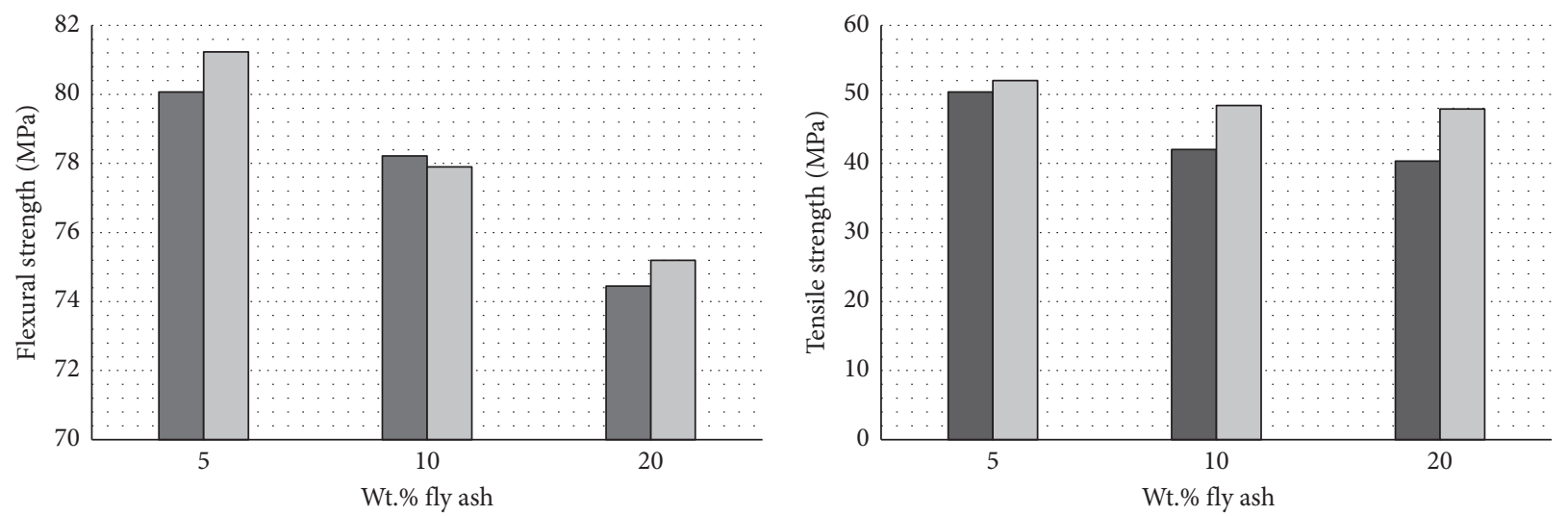

口 Fly ash

$\square$ Fly ash modified

(a)

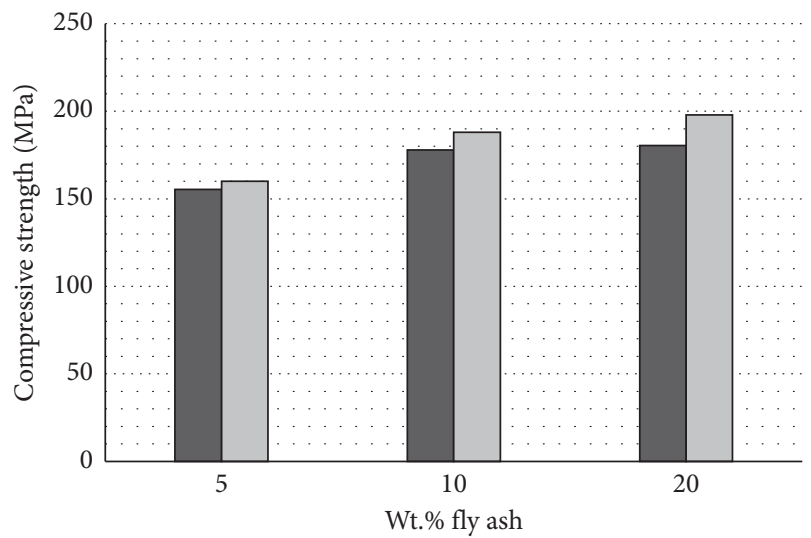

口 Fly ash

$\square$ Fly ash modified

Wt.\% fly ash

๘ Fly ash

$\square$ Fly ash modified

(b)

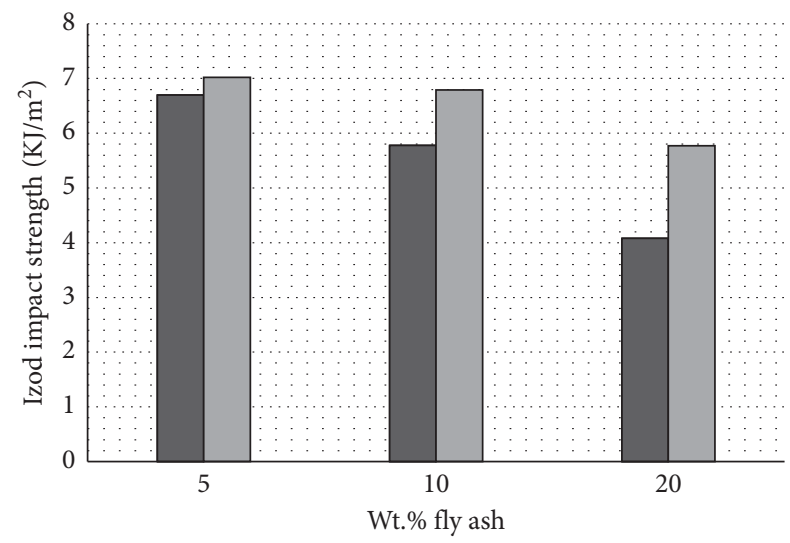

口 Fly ash

Fly ash modified

(c)

(d)

Figure 4: Effects on mechanical properties.

Based on the change of LOI in Figure 6, the initial resin sample with LOI of $23.2 \%$ is most flammable.

LOI of materials is increased from $21.4 \%$ to $23.2 \%$ corresponding to the amount of fly ash's increase from 0 to
20 wt.\%. A sample with 20 wt.\% of fly ash gaining LOI of $23.2 \%$ is the best flame retardant material.

The decrease in burning rate of the materials when increasing the amount of fly ash is shown in Figure 6. 


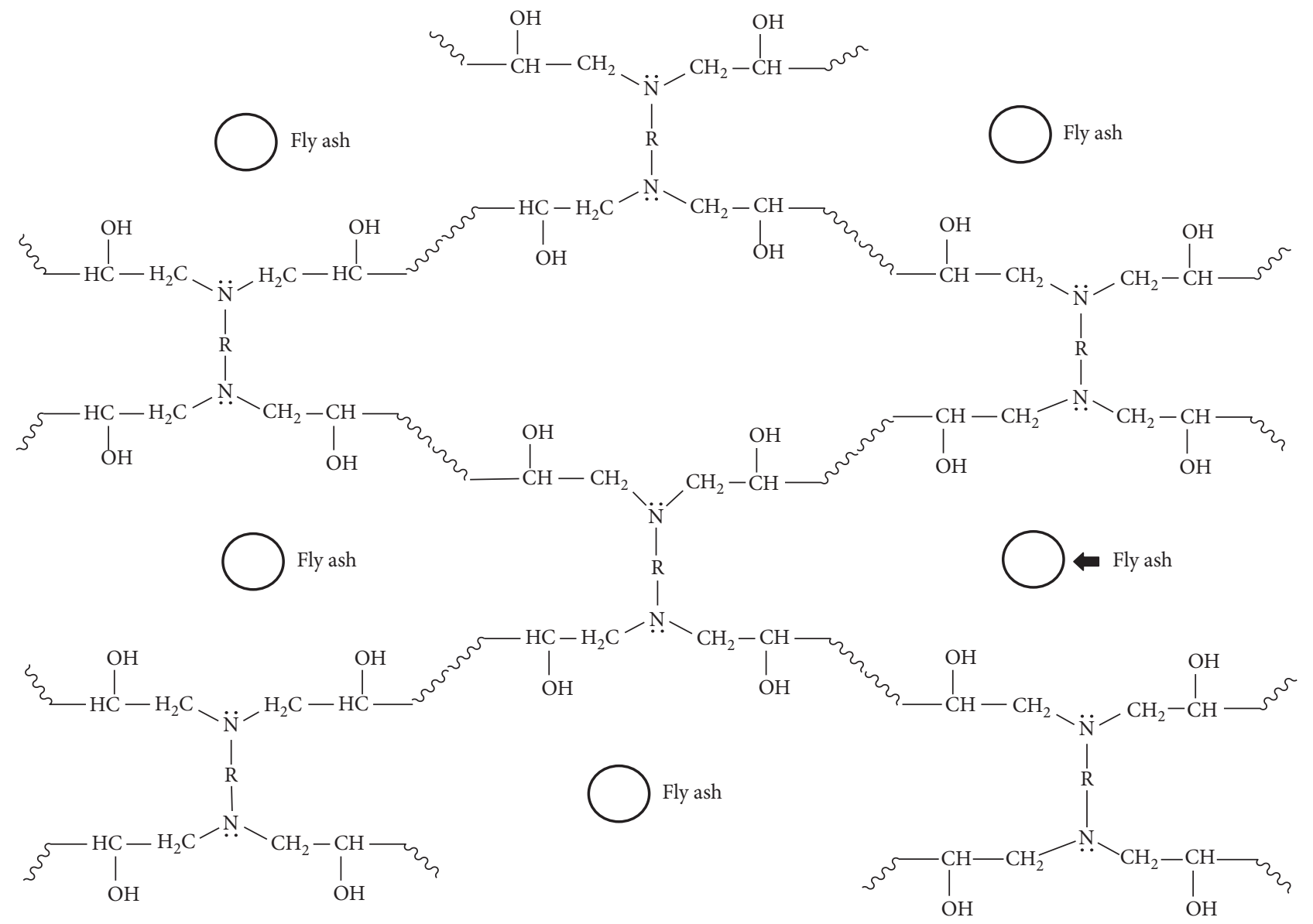

FIGURE 5: Images of fly ash particles alternating between epoxy molecules after plastic curing.

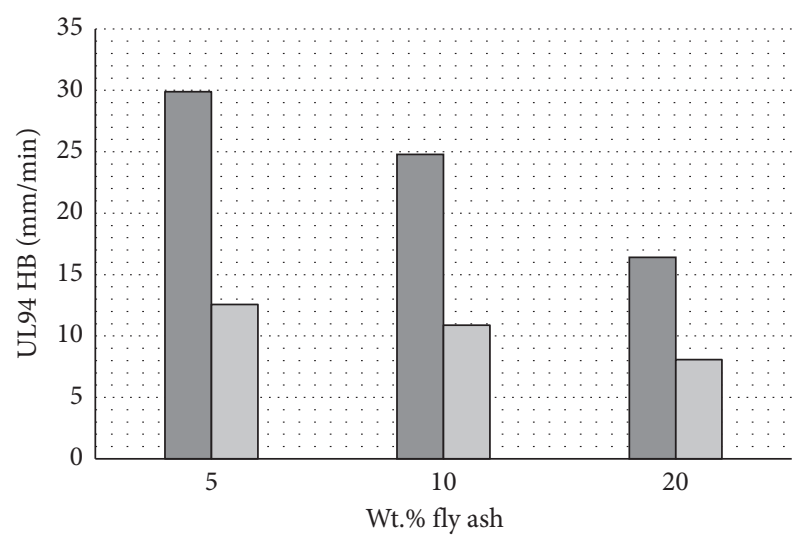

$\square$ Fly ash

$\square$ Fly ash modified

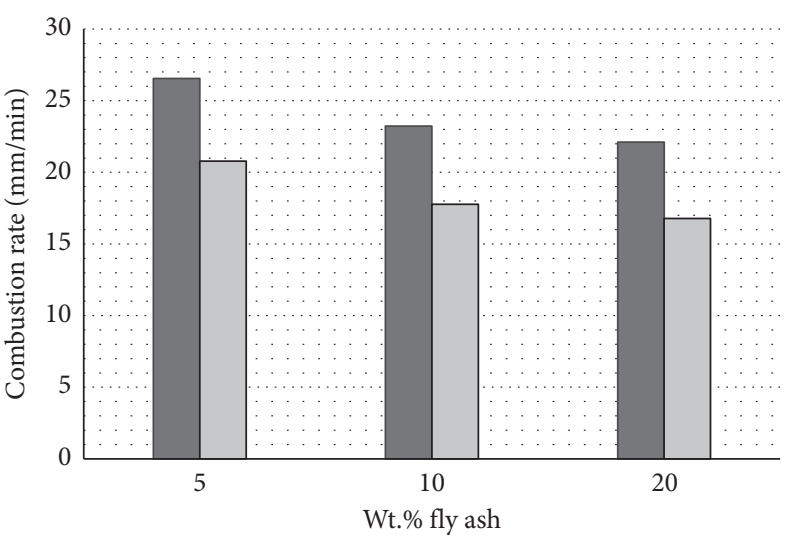

Gly ash

口 Fly ash modified

(a)

(b)

Figure 6: Continued. 


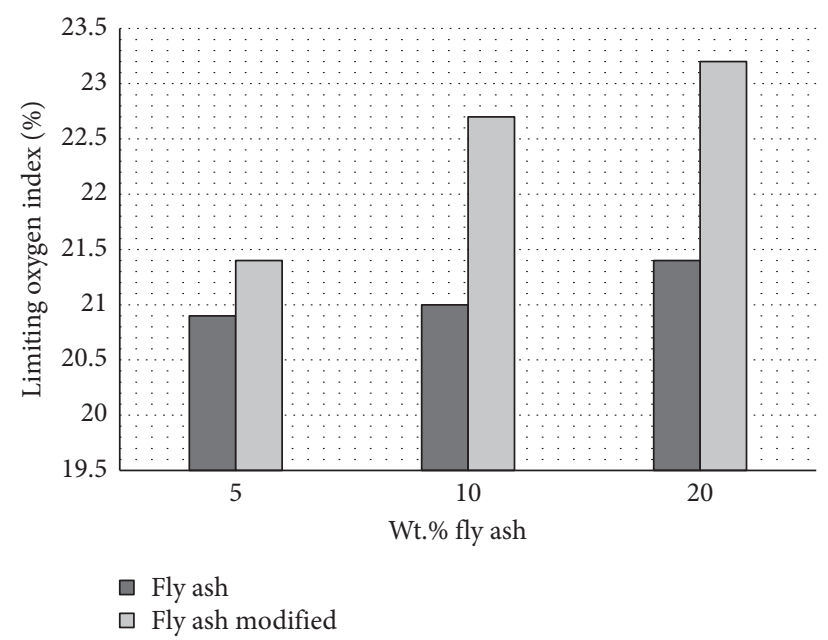

(c)

FIGURE 6: Effect of fly ash content on fire resistance of materials according to UL 94 HB standard; LOI index and combustion rate.

Without fly ash, samples' burning rate is $28.41 \mathrm{~mm} / \mathrm{min}$ in comparison to $16.28 \mathrm{~mm} / \mathrm{min}$ for sample added $20 \mathrm{wt} . \%$ of fly ash, proving the increase in flame retardant ability. Among all materials, the sample including $20 \mathrm{wt} . \%$ of fly ash is the best in flame retardant ability.

\section{Conclusions}

Epoxy Epikote 240 matrix composite was successfully prepared by using fly ash (FA). The interface between fly ash and epoxy matrix plays an important role in determining the mechanical strength of the composites. The global market consumption revealed that trends now are shifting towards ecofriendly FR due to the regulation imposed by authority, environment, safety, and health factors. This paper focuses on fly ash additive use in epoxy resin material. The fly ash exhibited compatibility as a coupling agent and enhanced mechanical properties and flame retardant properties.

\section{Data Availability}

The data used to support the findings of this study are available from the corresponding author upon request.

\section{Conflicts of Interest}

The authors declare that they have no conflicts of interest.

\section{Acknowledgments}

The authors wish to thank the Department of Chemical Technology, Hanoi University of Industry, Vietnam, for funding this work.

\section{References}

[1] Z. T. Yao, X. S. Ji, P. K. Sarker et al., "A comprehensive review on the applications of coal fly ash," Earth-Science Reviews, vol. 141, pp. 105-121, 2015.
[2] M. H. M. Ali, H. A. Rahman, S. H. Amirnordin, and N. A. Khan, "Eco-friendly flame-retardant additives for polyurethane foams: a short review," Key Engineering Materials, vol. 791, pp. 19-28, 2018.

[3] K. S. M. Kishore, S. M. Kulkarni, S. Sharathchandra, and D. Sunil, "On the use of an instrumented set-up to characterize the impact behaviour of an epoxy system containing varying fly ash content," Polymer Testing, vol. 21, no. 7, pp. 763-771, 2002.

[4] C. Vipulanandan and N. Dharmarajan, "Flexural behavior of polyester polymer concrete," Cement and Concrete Research, vol. 17 , no. 2, pp. 219-230, 1987.

[5] C. Vipulanandan, N. Dharmarajan, and E. Ching, "Mechanical behaviour of polymer concrete systems," Materials and Structures, vol. 21, no. 4, pp. 268-277, 2006.

[6] M. Singla and V. Chawla, "Mechanical properties of epoxy resin-fly ash composite," Journal of Minerals and Materials Characterization and Engineering, vol. 9, no. 3, pp. 199-210, 2010. 